\title{
Bifurcations in time-delay fully-connected networks with symmetry
}

\author{
Diego Paolo Ferruzzo Correa ${ }^{1, a}$ and José Roberto Castilho Piqueira ${ }^{1}$ \\ Universidade de São Paulo. \\ Escola Politécnica. \\ São Paulo, Brazil.
}

\begin{abstract}
In this work a brief method for finding steady-state and Hopf bifurcations in a $(R+1)$-th order $N$-node time-delay fully-connected network with symmetry is explored. A self-sustained Phase-Locked Loop is used as node. The irreducible representations found due to the network symmetry are used to find regions of time-delay independent stability/instability in the parameter space. Symmetry-preserving and symmetry-breaking bifurcations can be computed numerically using the $S_{n}$ map proposed in [1]. The analytic results show the existence of symmetry-breaking bifurcations with multiplicity $N-1$. A second-order $N$-node network is used as application example. This work is a generalization of some results presented in [2].
\end{abstract}

\section{Introduction}

Synchronization in coupled nonlinear oscillators with timedelay presents a great variety of interesting phenomena for many different areas in engineering, biology, chemistry, economy, etc. $[3,5,9,10,11,4,6]$. We study the effects of time-delay between weakly-coupled nodes in the synchronization, particularly how steady-state and Hopf bifurcations emerge when the time-delay is varied. For this purpose we shall choose a $(R+1)$-th order Phase-Locked Loop (PLL) as node, see [14], and a $N$-node time-delay fullyconnected network. The time-delay and the parameters in all nodes are considered identical. Our goal is to generalize some results proposed in [2] which are obtained for a second-order oscillator network.

\section{The Network model, symmetry and irreducible representations}

In the $(R+1)$-th order $N$-node fully connected network model with time-delay presented in [8] the phase detector output $v_{d}^{(i)}(t)$ is proportional to the weighted mean of all the phase detection related to the other $N-1$ outputs, thus

$$
\begin{aligned}
v_{d}^{(i)}(t)= & \frac{k_{m} V^{2}}{2(N-1)} \sum_{j=1, j \neq i}^{N-1}\left(\sin \left(\phi_{j}(t-\tau)-\phi_{i}(t)\right)\right. \\
& \left.+\sin \left(\phi_{j}(t-\tau)+\phi_{i}(t)\right)\right),
\end{aligned}
$$

here $\phi_{j}(t):=\theta_{j}(t)+\omega_{M} t$ is the so called full-phase, $\theta_{j}(t)$ is the instantaneous phase, $\omega_{M}, k_{m}$ and $V$ are real parameters and $\tau$ is the time-delay. The relationship between the phase detector output $v_{d}^{(i)}$ and the filter output $v_{c}^{(i)}$ is given by

$$
\mathcal{R}\left(v_{c}^{(i)}\right)=Q\left(v_{d}^{(i)}\right),
$$

the operators $\mathcal{R}(\cdot):=\sum_{r=0}^{R} \beta_{r} \frac{d^{r}}{d t^{r}}(\cdot)$ and $Q(\cdot):=\sum_{q=0}^{Q} \alpha_{q} \frac{d^{q}}{d t^{q}}(\cdot)$ are defined as in [8], provided $R>Q, \alpha_{q}, \beta_{r} \in \mathbb{R}$, and $\alpha_{0}, \beta_{0} \neq 0$, here without loss of generality we set $\beta_{R}=1$, for more details see $[14,15]$. The derivative of the instantaneous phase $\theta_{i}(t)$ is proportional to the filter output, thus $v_{c}^{(i)}(t)=\dot{\theta}_{i}(t) / k_{0}, k_{0}$ is a control real parameter, then $v_{c}^{(i)}=$ $\left(\dot{\phi}_{i}(t)-\omega_{M}\right) / k_{0}$, and by substituting into 1 we have

$$
\begin{aligned}
& \mathcal{R}\left(\dot{\phi}_{i}(t)\right)-\omega_{M} \beta_{0}= \\
& \frac{K}{N-1} Q\left(\sum_{j=1, \neq i}^{N-1} \sin \left(\phi_{j}(t-\tau)-\phi_{i}(t)\right)+\sin \left(\phi_{j}(t-\tau)+\phi_{i}(t)\right)\right),
\end{aligned}
$$

$K:=k_{0} k_{m} V^{2} / 2$. In [2] is given a proof of the $\mathbf{S}_{N^{-}}$-symmetry of 2 for the particular case $Q=0, R=1$ and $\alpha_{0}=$ $\beta_{0}$. The differential equation $\dot{X}(t)=F(X(t))$ on the phase space $\mathcal{X}=C\left([-\tau, 0], \mathbb{R}^{(R+1) N}\right)$ is equivariant with respect to the action of a Lie group $\Gamma$ on $\mathcal{X}$ such that $\gamma F(X)=$ $F(\gamma X)$ for all $X \in X, \gamma \in \Gamma$, see [16]. We write $\phi=$ $\left(\phi_{1}, \ldots, \phi_{N}\right)$, with $\phi_{j} \in C([-\tau, 0], \mathbb{R}), j=1, \ldots, N$, and let $x=\left(x^{(1)}, \ldots, x^{(N)}\right) \in \mathcal{X}$ where $x^{(i)}=\left(x_{1}^{(i)}, \ldots, x_{R+1}^{(i)}\right)$ and $x_{1}^{(i)}=\phi_{i}, x_{2}^{(i)}=\dot{\phi}_{i}, \ldots, x_{R+1}^{(i)}=\frac{d^{R}}{d t^{R}} \phi_{i}, i=1, \ldots, N$. If $x:[-\tau, A] \rightarrow \mathbb{R}^{n}$ is a continuous function with $A>0$ and if $t \in[0, A]$ then $X(t) \in C\left([-\tau, 0], \mathbb{R}^{n}\right)$ is defined by $X(t)(\theta)=x(t+\theta), \theta \in[-\tau, 0], t \in[0, A]$. Then 2 takes the form, see $[17,13]$

$$
\frac{\mathrm{d}}{\mathrm{d} t} X(t)=F(X(t), \eta)
$$

here $\eta=\left(\alpha_{0}, \ldots, \alpha_{Q}, \beta_{0}, \ldots, \beta_{R+1}, K, \omega_{M}, \tau\right) \in \mathbb{R}^{Q+R+1+3}$ is a parameter and $f=\left(f^{(1)}, \ldots, f^{(N)}\right)$ is such that 2 can also be rewritten as autonomous nonlinear delay differential equation (DDE),

\footnotetext{
a e-mail: dferruzzo@usp.br
}

$$
\dot{x}(t)=f(x(t-\tau), x(t), \eta),
$$


i.e., $f^{(i)}=\left(f_{1}^{(i)}, \ldots, f_{R+1}^{(i)}\right)$, where $f_{k}^{(i)}=x_{k+1}^{(i)}, k=1, \ldots, R$, and

$$
\begin{aligned}
f_{R+1}^{(i)}= & -\sum_{j=2}^{R+1} \beta_{j-2} x_{j}^{(i)}+\omega_{M} \beta_{0} \\
& +\frac{K}{N-1} \sum_{j=1, j \neq i}^{N-1}\left[\alpha_{0}\left(\sin \left(x_{1 \tau}^{(j)}-x_{1}^{(i)}\right)+\sin \left(x_{1 \tau}^{(j)}+x_{1}^{(i)}\right)\right)\right. \\
& +\cos \left(x_{1 \tau}^{(j)}-x_{1}^{(i)}\right) \sum_{r=1}^{Q} \alpha_{r}\left(x_{(r+1) \tau}^{(j)}-x_{r+1}^{(i)}\right) \\
& \left.+\cos \left(x_{1 \tau}^{(j)}+x_{1}^{(i)}\right) \sum_{r=1}^{Q} \alpha_{r}\left(x_{(r+1) \tau}^{(j)}+x_{r+1}^{(i)}\right)+O\left(x_{\tau}^{(j)}, x^{(i)}\right)\right]
\end{aligned}
$$

the term $O\left(x_{\tau}^{(j)}, x^{(i)}\right)$ represents nonlinear higher order terms. In this point the proof of $\mathbf{S}_{N}$-symmetry of 4 is the same as given in [2] and it will be omitted here. The equilibria in 2 in this new coordinates become $x^{(i)}=\left(x_{1}^{ \pm}, 0, \ldots, 0\right)$ where $2 x_{1}^{+}=\arcsin \left(-\omega_{M} \beta_{0} / K \alpha_{0}\right)+2 k \pi$ and $2 x_{1}^{-}=\pi-$ $\arcsin \left(-\omega_{M} \beta_{0} / K \alpha_{0}\right)+2 k \pi$, with $k \in \mathbb{Z}$ and $\omega_{M} \beta_{0} / K \alpha_{0} \leq 1$. The equilibria are $\mathbf{S}_{N}$-invariant and also the linearization $A(\eta)=D F\left(x^{*}, \eta\right)$ with $F\left(x^{*}, \eta\right)$ as in 3 . The linear operator $L(\eta)$ associated to the linearization $A(\eta)$, restricted to the $i$-th node, see [12,17], is computed as in [2] The characteristic matrix $\Delta(\lambda ; \eta):=\lambda \mathrm{Id}-\mathrm{L}(\eta)$ has $N$ blocks $m_{\lambda}$ in its diagonal and blocks $m_{b}$ in all other entries, where

$$
\begin{aligned}
& m_{\lambda}=\left(\begin{array}{ccccc}
\lambda & -1 & 0 & \cdots & 0 \\
0 & \lambda & -1 & \cdots & 0 \\
\vdots & \vdots & \vdots & \ddots & \vdots \\
0 & 0 & 0 & \cdots & -1 \\
a_{1} & a_{2} & a_{3} & \cdots & \lambda+a_{R+1}
\end{array}\right), \\
& m_{b}=\left(\begin{array}{cccc|ccc}
0 & 0 & \cdots & 0 & 0 & \cdots & 0 \\
\vdots & \vdots & \ddots & \vdots & \vdots & \ddots & \vdots \\
b_{1} & b_{2} & \cdots & b_{Q+1} & 0 & \cdots & 0
\end{array}\right),
\end{aligned}
$$

and

$a_{j}=\left\{\begin{array}{cl}-K\left(\cos \left(2 x_{1}^{*}\right)-1\right) \alpha_{0} & , j=1 \\ \beta_{j-2}-K\left(\cos \left(2 x_{1}^{*}\right)-1\right) \alpha_{j-1} & , j=2, \ldots, Q+1, \\ \beta_{j-2} & , j=Q+2, \ldots, R+1 .\end{array}\right.$

$b_{j}=\left\{\begin{array}{cl}-\frac{K}{N-1}\left(\cos \left(2 x_{1}^{*}\right)+1\right) \alpha_{j-1} e^{-\lambda \tau}, j=1, \ldots, Q+1, \\ 0 & , j=Q+2, \ldots, R+1 .\end{array}\right.$

Due to the $\mathbf{S}_{N}$-symmetry the characteristic equation

$$
P(\lambda ; \eta):=\operatorname{det}(\Delta(\lambda ; \eta))=0
$$

decomposes into two irreducible representations, see [7], namely

$$
P(\lambda ; \eta)=P_{\mathrm{Fix}\left(\mathbf{S}_{\mathrm{N}}\right)}(\lambda ; \eta)\left(P_{X_{j}}(\lambda ; \eta)\right)^{N-1}=0,
$$

we identify the restriction of the characteristic function to the Fixed-point space and to the other complementary spaces as $P_{\mathrm{Fix}\left(\mathbf{S}_{\mathrm{N}}\right)}(\lambda ; \eta)=\operatorname{det}\left(\mathrm{m}_{\lambda}+(\mathrm{N}-1) \mathrm{m}_{\mathrm{b}}\right)=0$ and
$P_{X_{j}}(\lambda ; \eta)=\operatorname{det}\left(\mathrm{m}_{\lambda}-\mathrm{m}_{\mathrm{b}}\right)=0, j=1, \ldots, N-1$, respectively. In both the cases the characteristic transcendental functions have the form:

$$
P(\lambda ; \eta)=T(\lambda ; \xi)+S(\lambda ; \xi) e^{-\lambda \tau}=0,
$$

with parameter $\xi=\left(\alpha_{0}, \ldots, \alpha_{Q}, \beta_{0}, \ldots, \beta_{R+1}, K, \omega_{M}\right) . T(\cdot)$ and $S(\cdot)$ are polynomials in $\lambda$ with coefficients in $\mathbb{R}$. After some manipulations be obtain:

$$
\begin{aligned}
T(\lambda ; \xi)= & \lambda^{R+1}-K\left(\cos \left(2 x_{1}^{*}\right)-1\right) \sum_{j=1}^{Q+1} \alpha_{j-1} \lambda^{j-1} \\
& +\sum_{j=2}^{R+1} \beta_{j-2} \lambda^{j-1}, \\
S(\lambda ; \xi)= & \left\{\begin{array}{l}
-K\left(\cos \left(2 x_{1}^{*}\right)+1\right) \sum_{q=1}^{Q+1} \alpha_{q-1} \lambda^{q-1} \quad, \operatorname{Fix}\left(\mathbf{S}_{\mathrm{N}}\right) \\
+\frac{K}{N-1}\left(\cos \left(2 x_{1}^{*}\right)+1\right) \sum_{q=1}^{Q+1} \alpha_{q-1} \lambda^{q-1}, X_{j} .
\end{array}\right.
\end{aligned}
$$

The challenge of finding bifurcations in these two irreducible representations $P_{\mathrm{Fix}\left(\mathbf{S}_{N}\right)}$ and $P_{X_{j}}$ can be addressed numerically using the $S_{n}$ map proposed in [1]. As an example of analytical result we compute the conditions for timedelay independent stability/instability for a second order $N$-node fully connected network, thus the transcendental functions given in 5 and 6 with $R=1$ and $Q=0$ become:

$$
\begin{aligned}
P_{\mathrm{Fix}\left(\mathbf{S}_{\mathrm{N}}\right)}(\lambda ; \eta)= & \lambda^{2}+\beta_{0} \lambda+K \alpha_{0}\left(1-\cos \left(2 x_{1}^{*}\right)\right) \\
& -K \alpha_{0}\left(1+\cos \left(2 x_{1}^{*}\right)\right) e^{-\lambda \tau}=0 \\
P_{X_{j}}(\lambda ; \eta)= & \lambda^{2}+\beta_{0} \lambda+K \alpha_{0}\left(1-\cos \left(2 x_{1}^{*}\right)\right) \\
& +\frac{K}{N-1} \alpha_{0}\left(1+\cos \left(2 x_{1}^{*}\right)\right) e^{-\lambda \tau}=0 .
\end{aligned}
$$

It was proved in [2] that for this case the equilibrium $x_{1}^{-}$is stable independent of time-delay within the intersection of the curves

$$
\alpha_{0} K-\left(\beta_{0} / 2\right)^{2}<\omega_{M}^{2}, \quad \text { and } \quad \omega_{\mathrm{M}} \beta_{0} \leq \mathrm{K} \alpha_{0} \leq \frac{\beta_{0}^{2}}{2} .
$$

for $\alpha_{0}, \beta_{0}, K, \omega_{M} \in \mathbb{R}^{+}$.

\subsection{Conclusions}

Due to the symmetry of the network it is possible to find irreducible representations of lower order and on them to find preserving-symmetry and breaking symmetry bifurcations. Although preserving-symmetry bifurcations do not present multiplicity, breaking-symmetry bifurcations show $N-1$ multiplicity, this multiplicity is forced by the symmetry. In some cases it is possible to find analytical results related to the time-delay independent stability/instability. The study of stability of bifurcations in both irreducible representations will be the main focus in future research.

This study was carried out under support from Coorde nação de Aperfeiçoamento de Pessoal de Nivel Superior, CAPES - Brazil, and Conselho Nacional de Desenvolvimento Cientifico e Tecnológico, CNPq - Brazil. 


\section{References}

1. Beretta Edoardo and Kuang Yang. Geometric stability switch criteria in delay differential systems with delay dependent parameters. SIAM Journal on Mathematical Analysis, 33(5), (2002), 1144-1165.

2. Diego Paolo Ferruzzo Correa, Claudia Wulff, and Jose Roberto Castilho Piqueira. Symmetric bifurcation analysis of synchronous states of time-delayed coupled oscillators. (2013). http://arxiv.org/abs/1310.7014v5.

3. Rodrigo Carareto, Fernando Moya Orsatti, and Jos ÃC R.C. Piqueira. Reachability of the synchronous state in a mutually connected PLL network. AEU - International Journal of Electronics and Communications, 63(11), (2009), 986 - 991.

4. Maoyin Chen and Jürgen Kurths. Synchronization of time-delayed systems. Phys. Rev. E, 76:036212, (2007).

5. Diego Paolo F. Correa and José Roberto C. Piqueira. Synchronous states in time-delay coupled periodic oscillators: A stability criterion. Communications in Nonlinear Science and Numerical Simulation, 18(8), (2013), 2142 - 2152.

6. Mukeshwar Dhamala, Viktor K. Jirsa, and Mingzhou Ding. Enhancement of neural synchrony by time delay. Phys. Rev. Lett., 92:074104, Feb 2004.

7. Ana Paula S Dias and Ana Rodrigues. Hopf bifurcation with $\mathbf{S}_{N^{-}}$-symmetry. Nonlinearity, 22(3), (2009), 627.

8. Bueno Atila Madureira, Andre Alves Ferreira, and J. R. C. Piqueira. Fully connected PLL networks: How filter determines the number of nodes. Mathematical Problems in Engineering, (2009).

9. J. R. C. Piqueira, M. Q. Oliveira, and L. H. A. Monteiro. Synchronous state in a fully connected phaselocked loop network. Mathematical Problems in Engineering, (2006).

10. J. R. C. Piqueira. Network of phase-locking oscillators and a possible model for neural synchronization. Communications in Nonlinear Science and Numerical Simulation, 16(9), (2011), 3844 - 3854.

11. J. R. C. Piqueira, F.M. Orsatti, and L.H.A. Monteiro. Computing with phase locked loops: choosing gains and delays. Neural Networks, IEEE Transactions on, 14(1), (2003), $243-247$.

12. Jianhong Wu. Symmetry functional differential equations and neural networks with memory. Transactions of the American Mathematical Society, 350(12), (1998), 4799-4839.

13. Balakumar Balachandran, Tamás Kalmár-Nagy, and David E. Gilsinn, editors. Delay differential equations. (Springer, New York, 2009). Recent advances and new directions.

14. Roland Best. Phase Locked Loops: Design, Simulation, and Applications. (McGraw-Hill Professional, 2007).

15. Floyd M. Gardner. Phaselock Techniques. (John Wiley \& Sons, 2005).

16. Martin Golubitsky, Ian Stewart, and David G. Schaeffer. Singularities and groups in bifurcation theory. Vol. II, volume 69 of Applied Mathematical Sciences. (Springer-Verlag, New York, 1988).
17. Jack K. Hale. Theory of Functional Differential Equations (Applied Mathematical Sciences). (Springer, 1977). 